\title{
AN AGE-STRUCTURED MODEL FOR THE TRANSMISSION DYNAMICS OF HEPATITIS B*
}

\author{
LAN ZOU ${ }^{\dagger}$, SHIGUI RUAN ${ }^{\ddagger}$, AND WEINIAN ZHANG ${ }^{\dagger}$
}

\begin{abstract}
Hepatitis B virus (HBV) infection is endemic in many parts of the world. One of the characteristics of HBV transmission is the age structure of the host population. In this paper, we propose an age-structured model for the transmission dynamics of HBV. The host population is stratified by age and is divided into six subclasses: susceptible, latently infected, acutely infectious, carrier, recovered, and vaccinated individuals. By determining the basic reproduction number, we study the existence and stability of the disease-free and endemic steady state solutions of the model. Numerical simulations are performed to find optimal strategies for controlling the transmission of HBV.
\end{abstract}

Key words. HBV, age structure, steady state, stability, basic reproduction number

AMS subject classifications. 35Q92, 35B35, 92D30

DOI. $10.1137 / 090777645$

1. Introduction. Hepatitis $B$ virus (HBV) infection is widespread in many parts of the world, especially in Africa, Southeast Asia, the Middle East, South and Western Pacific islands, the interior Amazon River basin, and certain parts of the Caribbean (Centers for Disease Control and Prevention (CDC) [5]). By the estimation of the World Health Organization (WHO) [30], about 2 billion people have been infected with HBV. An estimate of 600,000 persons die each year due to the acute or chronic consequences of the virus [30]. HBV is the most common serious viral infection and a leading cause of death in China. Around 130 million people in China are carriers of HBV, almost a third of the people infected with HBV worldwide and about $10 \%$ of the general population in the country; among them, 30 million are chronically infected. Every year, 300,000 people die from HBV-related diseases in China, accounting for 40-50\% of HBV-related deaths worldwide (Jia and Zhuang [17], Liu et al. [19]). The specific character of $\mathrm{HBV}$ infection in China is that there is a very large number of HBV carriers.

Hepatitis B is transmitted through body fluids like blood, semen, and vaginal secretions. One of the most important factors influencing the probability of developing carriage of $\mathrm{HBV}$ is age. Acute HBV infection causes chronic (long-term) infection in $30-90 \%$ of persons infected as infants or young children and in less than $5 \%$ of adolescents and adults (Shepard et al. [26], Goldstein et al. [11]). There is no widely available effective treatment for chronic HBV carriers. Immunization against HBV is now considered a viable option. In 1991, WHO recommended that hepatitis B vaccination should be included in the national immunization program in countries with an HBsAg carrier prevalence of 8\% by 1995 and all countries by 1997 [29].

\footnotetext{
* Received by the editors November 18, 2009; accepted for publication (in revised form) August 25, 2010; published electronically November 4, 2010.

http://www.siam.org/journals/siap/70-8/77764.html

$\dagger$ Yangtze Center of Mathematics and Department of Mathematics, Sichuan University, Chengdu, Sichuan 610064, People's Republic of China (lanzou@yahoo.com.cn, matwuzhang@yahoo.com.cn). The first author's research was partially supported by the China Scholarship Council. The third author's research was partially supported by NSFC grant 10825104 and SRFDP grant 200806100002.

‡Department of Mathematics, University of Miami, Coral Gables, FL 33124-4250 (ruan@math. miami.edu). This author's research was partially supported by NSF grant DMS-1022728.
} 
In the last two decades, many mathematical models have been proposed to investigate the transmission dynamics of $\mathrm{HBV}$ in various countries and regions in the world. Anderson, May, and Nokes [2] described a model for the sexual transmission of $\mathrm{HBV}$, which includes heterogeneous mixing with respect to age and sexual activity. Edmunds et al. [9] illustrated the relation between the age at infection with HBV and the development of the carrier state. McLean and Blumberg [21] and Edmunds, Medley, and Nokes [7] studied models of HBV transmission in developing countries. Williams et al. [28] presented a model for HBV infection in the UK that takes into consideration differences of age and sex. Zhao, $\mathrm{Xu}$, and $\mathrm{Lu}$ [31] developed a mathematical model to predict the HBV transmission dynamics and to evaluate the long-term effectiveness of the vaccination program in China. Medley et al. [23] gave a model to show that the prevalence of infection is determined by a feedback mechanism that relates the rate of transmission, average age at infection, and age-related probability of developing carriage following infection.

The age structure of a population has been regarded as an important factor for the dynamics of disease transmission since the work of Sharpe and Lotka [25] and McKendrick [22]. Great effort has been devoted to determining the threshold conditions for the disease to become endemic, the stability of steady state solutions, and the global behavior of age-structured epidemic models; we refer to Anderson and May [1], Busenberg, Cooke, and Iannelli [3], Busenberg, Iannelli, and Thieme [4], Feng, Huang, and Castillo-Chavez [10], Greenhalgh [12, 13], Iannelli [14], Inaba [15, 16], Li, Gupur, and Zhu [18], Martcheva and Crispino-O'Connell [20], Webb [27], and the references cited therein. Age-structured models have also been used to model the transmission dynamics of HBV by some researchers; see Edmunds et al. [9], McLean and Blumberg [21], Zhao, Xu, and $\mathrm{Lu}$ [31], etc.

In a previous study (Zou, Zhang, and Ruan [32]), we developed a mathematical model described by ordinary differential equations to study the transmission dynamics and control of HBV in mainland China, taking account of the specific character of the virus infection in the country. We analyzed the existence and stability of the disease-free and disease-endemic steady states of the model and used the model to simulate the HBV data reported by the Ministry of Health of China (MOHC) [24].

Based on our previous work [32], in this article we propose an age-structured model for the transmission of HBV. We qualitatively analyze the model, including the existence of positive solutions, and the stability and existence of the disease-free and disease-endemic steady states in terms of the basic reproduction number. Some numerical simulations of the model are also given to illustrate the results and to find optimal strategies in controlling HBV infection.

The paper is organized as follows. In section 2 , we present the mathematical model for the transmission dynamics of hepatitis B. The existence of positive solutions is considered in section 3 . Section 4 deals with the existence and stability of the diseasefree steady state. In section 5 , we give conditions that guarantee the existence and stability of disease-endemic steady states. In section 6 , some numerical simulations of the model are presented. The paper ends with a brief discussion in section 7 .

2. The model. We propose an age-structured model to study the transmission dynamics of HBV. As the model includes age-dependent processes such as the force of infection and the probability of developing the carrier state, the host population is stratified by age. We divide the total population of size $N(a, t)$ into six subclasses: susceptible $S(a, t)$, latently infected $L(a, t)$, acutely infectious $I(a, t)$, carrier $C(a, t)$, recovered $R(a, t)$, and vaccinated $V(a, t)$, with age distribution at time $t$. The vari- 


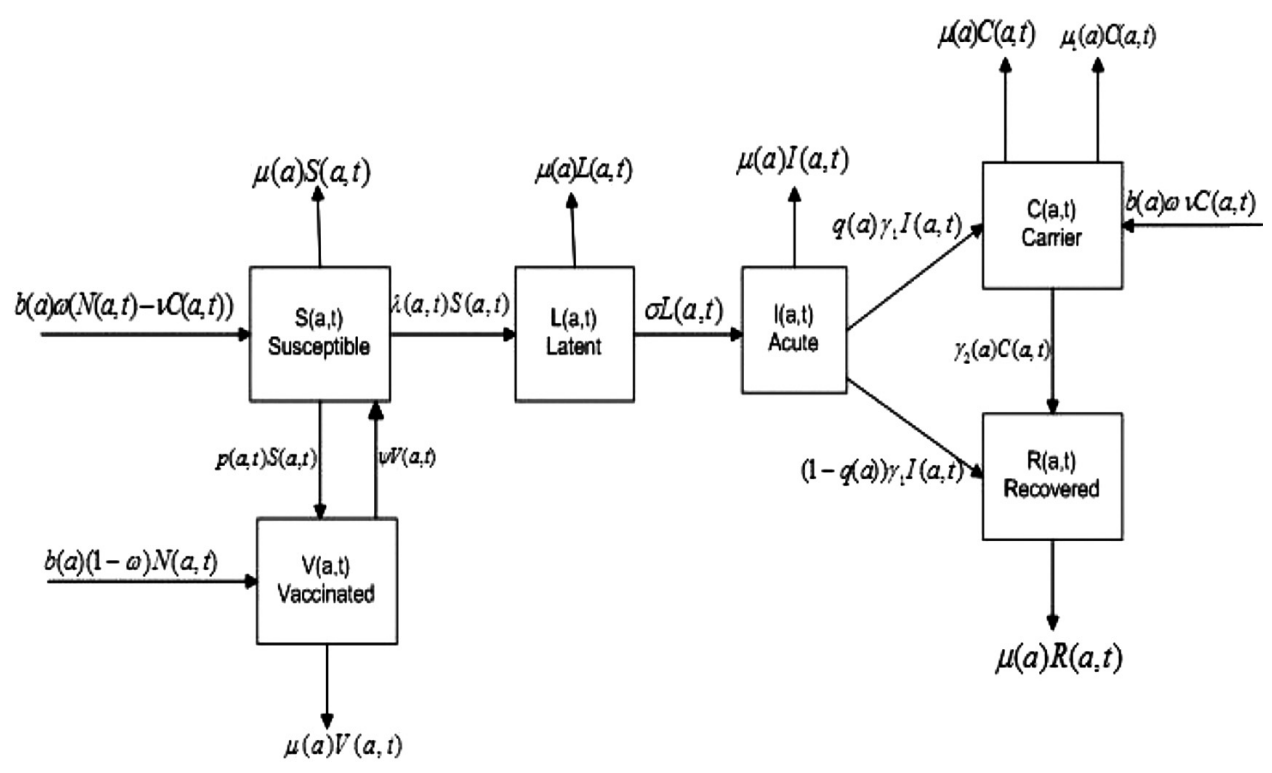

FIG. 1. Flowchart of HBV transmission in a population.

ables and model structure are described in Figure 1.

The age-structured model for the transmission of HBV is described by the following system of partial differential equations:

$$
\begin{aligned}
& \frac{\partial S}{\partial a}+\frac{\partial S}{\partial t}=b(a) \omega(N(a, t)-\nu C(a, t))+\psi V(a, t)-(\mu(a)+\lambda(a, t)+p(a, t)) S(a, t) \\
& \frac{\partial L}{\partial a}+\frac{\partial L}{\partial t}=\lambda(a, t) S(a, t)-(\mu(a)+\sigma) L(a, t) \\
& \frac{\partial I}{\partial a}+\frac{\partial I}{\partial t}=\sigma L(a, t)-\left(\mu(a)+\gamma_{1}\right) I(a, t) \\
& \frac{\partial C}{\partial a}+\frac{\partial C}{\partial t}=b(a) \omega \nu C(a, t)+q(a) \gamma_{1} I(a, t)-\left(\mu(a)+\mu_{1}(a)+\gamma_{2}(a)\right) C(a, t) \\
& \frac{\partial R}{\partial a}+\frac{\partial R}{\partial t}=\gamma_{2}(a) C(a, t)+(1-q(a)) \gamma_{1} I(a, t)-\mu(a) R(a, t) \\
& \frac{\partial V}{\partial a}+\frac{\partial V}{\partial t}=b(a)(1-\omega) N(a, t)+p(a, t) S(a, t)-(\psi+\mu(a)) V(a, t)
\end{aligned}
$$

with initial and boundary conditions

$$
\begin{aligned}
S(0, t) & =\int_{a_{1}}^{a_{2}} b(a) \omega[S(a, t)+L(a, t)+I(a, t)+R(a, t)+V(a, t)+(1-\nu) C(a, t)] d a, \\
C(0, t) & =\int_{a_{1}}^{a_{2}} b(a) \omega \nu C(a, t) d a, \\
V(0, t) & =\int_{a_{1}}^{a_{2}} b(a)(1-\omega)[S(a, t)+L(a, t)+I(a, t)+C(a, t)+R(a, t)+V(a, t)] d a, \\
L(0, t) & =I(0, t)=R(0, t)=0, \\
S(a, 0) & =S_{0}(a), L(a, 0)=L_{0}(a), I(a, 0)=I_{0}(a), C(a, 0)=C_{0}(a), \\
R(a, 0) & =R_{0}(a), V(a, 0)=V_{0}(a),
\end{aligned}
$$

Copyright (c) by SIAM. Unauthorized reproduction of this article is prohibited. 
where

$$
\lambda(a, t)=\int_{0}^{a_{+}} \beta\left(a, a^{\prime}\right) \frac{I\left(a^{\prime}, t\right)+\epsilon C\left(a^{\prime}, t\right)}{N\left(a^{\prime}, t\right)} d a^{\prime},
$$

$a_{+}>0$ is the maximum age of an individual, and the interval $\left(a_{1}, a_{2}\right)$ represents the female fertile period. Then we obtain the following equations for the total population $N(a, t)$ :

$$
\begin{aligned}
& \frac{\partial N(a, t)}{\partial a}+\frac{\partial N(a, t)}{\partial t}=(b(a)-\mu(a)) N(a, t)-\mu_{1}(a) C(a, t), \\
& N(0, t)=\int_{a_{1}}^{a_{2}} b(a) N(a, t) d a, \quad N(a, 0)=N_{0}(a) .
\end{aligned}
$$

The parameters are described as follows:

$b(a)$ - birth rate

$\mu(a)$ - natural mortality rate

$\mu_{1}(a)$ - HBV-related mortality rate

$\beta\left(a, a^{\prime}\right)$ - probability that an infective individual of age $a^{\prime}$ will have contact with and successfully infect a susceptible individual of age $a$

$\epsilon$ - reduced transmission rate from chronic carriers compared to acute infections

$\sigma$ - rate moving from latent to acute

$\gamma_{1}$ - rate moving from acute to carrier

$\gamma_{2}(a)$ - rate moving from carrier to vaccinated

$p(a, t)$ - vaccination rate against $\mathrm{HBV}$

$(1-\omega)$ - proportion of births with successful vaccination

$q(a)$ - probability an individual fails to clear an acute infection and develops to carrier state

$\psi$ - rate of waning vaccine-induced immunity

$\nu$ - proportion of perinatally infected (from carrier mothers).

Let

$$
\begin{aligned}
& s(a, t)=\frac{S(a, t)}{N(a, t)}, \quad \ell(a, t)=\frac{L(a, t)}{N(a, t)}, \quad x(a, t)=\frac{I(a, t)}{N(a, t)}, \\
& c(a, t)=\frac{C(a, t)}{N(a, t)}, \quad v(a, t)=\frac{V(a, t)}{N(a, t)}, \quad r(a, t)=\frac{R(a, t)}{N(a, t)} .
\end{aligned}
$$

Let $k(a)$ be the age-specific (average) probability of becoming infected through contact with infectious individuals, and let $\tilde{\beta}\left(a^{\prime}\right)$ be the age-specific per-capita contact/ activity rate. Following the discussion of infection coefficients in Anderson and May [1], we make the following assumption.

Assumption 2.1. Assume that

$$
\beta\left(a, a^{\prime}\right)=k(a) \tilde{\beta}\left(a^{\prime}\right) .
$$

Under the above assumption, system (2.1) can be normalized as the following 
system:

$$
\begin{aligned}
& \frac{\partial s}{\partial a}+\frac{\partial s}{\partial t}= b(a) \omega(1-\nu c(a, t))+\psi v(a, t)-\left(b(a)-\mu_{1}(a) c(a, t)\right) s(a, t) \\
&-(\lambda(a, t)+p(a, t)) s(a, t), \\
& \frac{\partial \ell}{\partial a}+\frac{\partial \ell}{\partial t}=\lambda(a, t) s(a, t)-\left(\sigma+b(a)-\mu_{1}(a) c(a, t)\right) \ell(a, t), \\
& \frac{\partial x}{\partial a}+\frac{\partial x}{\partial t}=\sigma \ell(a, t)-\left(\gamma_{1}+b(a)-\mu_{1}(a) c(a, t)\right) x(a, t), \\
& \frac{\partial c}{\partial a}+\frac{\partial c}{\partial t}=b(a) \omega \nu c(a, t)+q(a) \gamma_{1} x(a, t)-\left(\mu_{1}(a)(1-c(a, t))+b(a)+\gamma_{2}(a)\right) c(a, t), \\
& \frac{\partial r}{\partial a}+\frac{\partial r}{\partial t}=\gamma_{2}(a) c(a, t)+(1-q(a)) \gamma_{1} x(a, t)-\left(b(a)-\mu_{1}(a) c(a, t)\right) r(a, t), \\
& \frac{\partial v}{\partial a}+\frac{\partial v}{\partial t}=b(a)(1-\omega)+p(a, t) s(a, t)-\left(\psi+b(a)-\mu_{1}(a) c(a, t)\right) v(a, t),
\end{aligned}
$$

with boundary conditions

$$
\begin{array}{rlrl}
s(0, t)=\omega\left(1-\int_{a_{1}}^{a_{2}} \nu c(a, t) d a\right), & c(0, t) & =\int_{a_{1}}^{a_{2}} \omega \nu c(a, t) d a, \\
v(0, t)=1-\omega, & \ell(0, t)=x(0, t)=r(0, t)=0,
\end{array}
$$

where

$$
\lambda(a, t)=k(a) \int_{0}^{a_{+}} \tilde{\beta}(a)(x(a, t)+\epsilon c(a, t)) d a .
$$

In the following, we consider system (2.5) with initial conditions

$$
\begin{array}{lll}
s(a, 0)=s_{0}(a), & \ell(a, 0)=\ell_{0}(a), & x(a, 0)=x_{0}(a), \\
c(a, 0)=c_{0}(a), & r(a, 0)=r_{0}(a), & v(a, 0)=v_{0}(a) .
\end{array}
$$

3. Existence of positive solutions. Since $r(a, t)$ can be obtained by

$$
r(a, t)=1-s(a, t)-\ell(a, t)-x(a, t)-c(a, t)-v(a, t),
$$

it suffices to discuss the system in terms of only $s(a, t), \ell(a, t), x(a, t), c(a, t)$, and $v(a, t)$. Since in a short period of time the change of $p$ is small, we assume that the vaccination rate $p(a, t)=p(a)$ depends only on the age $a$. Thus, we obtain the following system:

$$
\begin{aligned}
& \frac{\partial s}{\partial a}+\frac{\partial s}{\partial t}= b(a) \omega(1-\nu c(a, t))+\psi v(a, t)-\left(b(a)-\mu_{1}(a) c(a, t)\right) s(a, t) \\
&-(\lambda(a, t)+p(a)) s(a, t), \\
& \frac{\partial \ell}{\partial a}+\frac{\partial \ell}{\partial t}=\lambda(a, t) s(a, t)-\left(\sigma+b(a)-\mu_{1}(a) c(a, t)\right) \ell(a, t), \\
& \frac{\partial x}{\partial a}+\frac{\partial x}{\partial t}=\sigma \ell(a, t)-\left(\gamma_{1}+b(a)-\mu_{1}(a) c(a, t)\right) x(a, t), \\
& \frac{\partial c}{\partial a}+\frac{\partial c}{\partial t}=b(a) \omega \nu c(a, t)+q(a) \gamma_{1} x(a, t)-\left(\mu_{1}(a)(1-c(a, t))+b(a)+\gamma_{2}(a)\right) c(a, t), \\
& \frac{\partial v}{\partial a}+\frac{\partial v}{\partial t}=b(a)(1-\omega)+p(a) s(a, t)-\left(\psi+b(a)-\mu_{1}(a) c(a, t)\right) v(a, t),
\end{aligned}
$$

Copyright (c) by SIAM. Unauthorized reproduction of this article is prohibited. 
with boundary conditions

$$
\begin{array}{rlrl}
s(0, t)=\omega\left(1-\int_{a_{1}}^{a_{2}} \nu c(a, t) d a\right), & & c(0, t)=\int_{a_{1}}^{a_{2}} \omega \nu c(a, t) d a, \\
v(0, t)=1-\omega, & \ell(0, t)=x(0, t)=0 .
\end{array}
$$

Consider the Banach space

$$
\mathbf{X}=L^{1}\left(0, a_{+}\right) \times L^{1}\left(0, a_{+}\right) \times L^{1}\left(0, a_{+}\right) \times L^{1}\left(0, a_{+}\right) \times L^{1}\left(0, a_{+}\right)
$$

endowed with the norm

$$
\|\phi\|=\sum_{i=1}^{5}\left\|\phi_{i}\right\| \text { for } \phi(a)=\left(\phi_{1}(a), \phi_{2}(a), \phi_{3}(a), \phi_{4}(a), \phi_{5}(a)\right)^{T} \in \mathbf{X},
$$

where $\|\cdot\|$ is the norm of $L^{1}\left(0, a_{+}\right)$. The state space of system (3.1) is

$$
\Omega:=\left\{(s, l, x, c, v) \in \mathbf{X}_{+} \mid 0 \leq s+\ell+x+c+v \leq 1\right\},
$$

where $\mathbf{X}_{+}=L_{+}^{1}\left(0, a_{+}\right) \times L_{+}^{1}\left(0, a_{+}\right) \times L_{+}^{1}\left(0, a_{+}\right) \times L_{+}^{1}\left(0, a_{+}\right) \times L_{+}^{1}\left(0, a_{+}\right)$, and $L_{+}^{1}\left(0, a_{+}\right)$ denotes the positive cone of $L^{1}\left(0, a_{+}\right)$.

Let $A$ be a linear operator defined by

$$
(A \phi)(a)=\left(A_{1}, A_{2}, A_{3}, A_{4}, A_{5}\right)^{T},
$$

where

$$
\begin{aligned}
& A_{1}=\left(-\frac{d \phi_{1}}{d a}-(b(a)+p(a)) \phi_{1}, 0,0,-b(a) \omega \nu \phi_{4}, \psi \phi_{5}\right), \\
& A_{2}=\left(0,-\frac{d \phi_{2}}{d a}-(\sigma+b(a)) \phi_{2}, 0,0,0\right), \\
& A_{3}=\left(0, \sigma \phi_{2},-\frac{d \phi_{3}}{d a}-\left(\gamma_{1}+b(a)\right) \phi_{3}, 0,0\right), \\
& A_{4}=\left(0,0, q(a) \gamma_{1} \phi_{3},-\frac{d \phi_{4}}{d a}-\left(\mu_{1}(a)+\gamma_{2}(a)-b(a) \omega \nu\right) \phi_{4}, 0\right), \\
& A_{5}=\left(p(a) \phi_{1}, 0,0,0,-\frac{d \phi_{5}}{d a}-(\psi+b(a)) \phi_{5}\right), \\
& \phi(a)=\left(\phi_{1}(a), \phi_{2}(a), \phi_{3}(a), \phi_{4}(a), \phi_{5}(a)\right)^{T} \in D(A),
\end{aligned}
$$

and the domain $D(A)$ is given as

$$
\begin{aligned}
& D(A)=\left\{\phi \in \mathbf{X} \mid \phi_{i} \in A C\left[0, a_{+}\right),\right. \\
&\left.\phi(0)=\left(\omega\left(1-\int_{a_{1}}^{a_{2}} \nu \phi_{3}(a) d a\right), 0, \omega \nu \int_{a_{1}}^{a_{2}} \phi_{3}(a) d a, 0,1-\omega\right)^{T}\right\},
\end{aligned}
$$

where $A C\left[0, a_{+}\right)$denotes the set of absolutely continuous functions on $\left[0, a_{+}\right)$.

We also define a nonlinear operator $F: \mathbf{X} \rightarrow \mathbf{X}$ by

$$
(F \phi)(a)=\left(\begin{array}{c}
b(a) \omega+\mu_{1}(a) \phi_{4} \phi_{1}-\left(\left(Q \phi_{3}\right)(a)+\epsilon\left(Q \phi_{4}\right)(a)\right) \phi_{1} \\
\left(\left(Q \phi_{3}\right)(a)+\epsilon\left(Q \phi_{4}\right)(a)\right) \phi_{1}+\mu_{1}(a) \phi_{4} \phi_{2} \\
\mu_{1}(a) \phi_{4} \phi_{3} \\
\mu_{1}(a) \phi_{4}^{2} \\
b(a)(1-\omega)+\mu_{1}(a) \phi_{4} \phi_{5}
\end{array}\right),
$$

Copyright $@$ by SIAM. Unauthorized reproduction of this article is prohibited. 
where $Q$ is a bounded linear operator on $L^{1}\left(0, a_{+}\right)$given by

$$
(Q f)(a)=\int_{0}^{a_{+}} \beta\left(a, a^{\prime}\right) f\left(a^{\prime}\right) d a^{\prime} .
$$

Let $u(t)=(s(\cdot, t), \ell(\cdot, t), x(\cdot, t), c(\cdot, t), v(\cdot, t))$. Thus, we can rewrite the system as an abstract Cauchy problem

$$
\frac{d u(t)}{d t}=A u(t)+F(u(t)), \quad u(0)=u_{0} \in \mathbf{X},
$$

where $u_{0}(a)=\left(s_{0}(a), \ell_{0}(a), x_{0}(a), c_{0}(a), v_{0}(a)\right)^{T}$.

For $A$ and $F$, following Inaba [16] and Webb [27], we have the following results.

Lemma 3.1. The operator $A$ generates a $C_{0}$ semigroup $e^{t A}$ and the space $\Omega$ is positively invariant with respect to the semiflow defined by $e^{t A}$.

Lemma 3.2. The operator $F$ is continuously Fréchet differentiable on $\mathbf{X}$.

TheOREM 3.3. For each $u_{0} \in \mathbf{X}_{+}$, there are a maximal interval of existence $\left[0, t_{0}\right)$ and a unique continuous mild solution $u\left(t, u_{0}\right) \in \mathbf{X}_{+}, t \in\left[0, t_{0}\right)$ for (3.4) such that

$$
u(t)=e^{A t} u_{0}+\int_{0}^{t} e^{A(t-\tau)} F(u(\tau)) d \tau .
$$

4. The disease-free steady state. A steady state $(s(a), \ell(a), x(a), c(a), v(a))$ of system (3.1) must satisfy the following time-independent system of ordinary differential equations:

$$
\begin{aligned}
& \frac{d s}{d a}=b(a) \omega(1-\nu c(a))+\psi v(a)-\left(b(a)-\mu_{1}(a) c(a)\right) s(a)-(\lambda(a)+p(a)) s(a), \\
& \frac{d \ell}{d a}=\lambda(a) s(a)-\left(\sigma+b(a)-\mu_{1}(a) c(a)\right) \ell(a), \\
& \frac{d x}{d a}=\sigma \ell(a)-\left(\gamma_{1}+b(a)-\mu_{1}(a) c(a)\right) x(a), \\
& \frac{d c}{d a}=b(a) \omega \nu c(a)+q(a) \gamma_{1} x(a)-\left(\mu_{1}(a)(1-c(a))+b(a)+\gamma_{2}(a)\right) c(a), \\
& \frac{d v}{d a}=b(a)(1-\omega)+p(a) s(a)-\left(\psi+b(a)-\mu_{1}(a) c(a)\right) v(a),
\end{aligned}
$$

with initial value conditions

$s(0)=\omega\left(1-\int_{a_{1}}^{a_{2}} \nu c(a) d a\right), \quad c(0)=\int_{a_{1}}^{a_{2}} \omega \nu c(a) d a, \quad v(0)=1-\omega, \quad \ell(0)=x(0)=0$,

where

$$
\lambda(a)=k(a) \int_{0}^{a_{+}} \tilde{\beta}(a)(x(a)+\epsilon c(a)) d a .
$$

Therefore, we obtain the disease-free steady state

$$
E^{0}=\left(s^{0}(a), \ell^{0}(a), x^{0}(a), c^{0}(a), v^{0}(a)\right),
$$

where

$$
\begin{aligned}
& s^{0}(a)=\omega e^{-\psi a-\int_{0}^{a}(b(\xi)+p(\xi)) d \xi}+\int_{0}^{a} e^{-\psi(a-\eta)-\int_{\eta}^{a}(b(\xi)+p(\xi)) d \xi}(b(\eta) \omega+\psi) d \eta, \\
& \ell^{0}(a)=x^{0}(a)=c^{0}(a)=0, \quad v^{0}(a)=1-s^{0}(a) .
\end{aligned}
$$

Copyright $@$ by SIAM. Unauthorized reproduction of this article is prohibited. 
To study the stability of the disease-free steady state $E^{0}$, we denote the perturbations by $\bar{s}(a, t), \bar{\ell}(a, t), \bar{x}(a, t), \bar{c}(a, t), \bar{v}(a, t)$, respectively. The perturbations satisfy the following equations:

$$
\begin{aligned}
& \frac{\partial \bar{s}}{\partial a}+\frac{\partial \bar{s}}{\partial t}=(\psi-b(a) \omega \nu) \bar{c}(a, t)-(b(a)+p(a)) \bar{s}(a, t)+\left(\mu_{1}(a) \bar{c}(a, t)-\bar{\lambda}(a, t)\right) s^{0}(a), \\
& \frac{\partial \bar{\ell}}{\partial a}+\frac{\partial \bar{\ell}}{\partial t}=\bar{\lambda}(a, t) s^{0}(a)-(\sigma+b(a)) \bar{\ell}(a, t) \\
& \frac{\partial \bar{x}}{\partial a}+\frac{\partial \bar{x}}{\partial t}=\sigma \bar{\ell}(a, t)-\left(\gamma_{1}+b(a)\right) \bar{x}(a, t), \\
& \frac{\partial \bar{c}}{\partial a}+\frac{\partial \bar{c}}{\partial t}=b(a) \omega \nu \bar{c}(a, t)+q(a) \gamma_{1} \bar{x}(a, t)-\left(\mu_{1}(a)+b(a)+\gamma_{2}(a)\right) \bar{c}(a, t), \\
& \frac{\partial \bar{v}}{\partial a}+\frac{\partial \bar{v}}{\partial t}=p(a) \bar{s}(a, t)-(\psi+b(a)) \bar{v}(a, t)+\mu_{1}(a) \bar{c}(a, t) v^{0}(a)
\end{aligned}
$$

with boundary conditions

$$
\begin{aligned}
& \bar{s}(0, t)=-\omega \int_{a_{1}}^{a_{2}} \nu \bar{c}(a, t) d a, \quad \bar{c}(0, t)=\int_{a_{1}}^{a_{2}} \omega \nu \bar{c}(a, t) d a, \\
& \bar{\ell}(0, t)=\bar{x}(0, t)=\bar{v}(0, t)=0
\end{aligned}
$$

where $\bar{\lambda}(a, t)=k(a) \int_{0}^{a_{+}} \tilde{\beta}(a)(\bar{x}(a, t)+\epsilon \bar{c}(a, t)) d a$.

Now, we consider the exponential solutions of system (4.2) of the form

$$
\begin{array}{ll}
\bar{s}(a, t)=\bar{s}(a) e^{\varpi t}, & \bar{\ell}(a, t)=\bar{\ell}(a) e^{\varpi t}, \quad \bar{x}(a, t)=\bar{x}(a) e^{\varpi t}, \\
\bar{c}(a, t)=\bar{c}(a) e^{\varpi t}, & \bar{v}(a, t)=\bar{v}(a) e^{\varpi t},
\end{array}
$$

where $\bar{s}(a), \bar{\ell}(a), \bar{x}(a), \bar{c}(a), \bar{v}(a)$, and the growth exponent $\varpi$ satisfy the following equations:

$$
\begin{aligned}
& \frac{d \bar{s}}{d a}=-\varpi \bar{s}(a)+(\psi-b(a) \omega \nu) \bar{c}(a)-(b(a)+p(a)) \bar{s}(a)+\left(\mu_{1}(a) \bar{c}(a)-\hat{\lambda}(a)\right) s^{0}(a), \\
& \frac{d \bar{\ell}}{d a}=-\varpi \bar{\ell}(a)+\hat{\lambda}(a) s^{0}(a)-(\sigma+b(a)) \bar{\ell}(a) \\
& \frac{d \bar{x}}{d a}=-\varpi \bar{x}(a)+\sigma \bar{\ell}(a)-\left(\gamma_{1}+b(a)\right) \bar{x}(a) \\
& \frac{d \bar{c}}{d a}=-\varpi \bar{c}(a)+b(a) \omega \nu \bar{c}(a)+q(a) \gamma_{1} \bar{x}(a)-\left(\mu_{1}(a)+b(a)+\gamma_{2}(a)\right) \bar{c}(a), \\
& \frac{d \bar{v}}{d a}=-\varpi \bar{v}(a)+p(a) \bar{s}(a)-(\psi+b(a)) \bar{v}(a)+\mu_{1}(a) \bar{c}(a) v^{0}(a)
\end{aligned}
$$

with boundary conditions

$$
\bar{s}(0)=-\omega \int_{a_{1}}^{a_{2}} \nu \bar{c}(a) d a, \quad \bar{c}(0)=\int_{a_{1}}^{a_{2}} \omega \nu \bar{c}(a) d a, \quad \bar{\ell}(0)=\bar{x}(0)=\bar{v}(0)=0,
$$

where

$$
\hat{\lambda}(a)=k(a) \int_{0}^{a_{+}} \tilde{\beta}\left(a^{\prime}\right)\left(\bar{x}\left(a^{\prime}\right)+\epsilon \bar{c}\left(a^{\prime}\right)\right) d a^{\prime} .
$$

Copyright $@$ by SIAM. Unauthorized reproduction of this article is prohibited. 
Let $\Lambda=\int_{0}^{a_{+}} \tilde{\beta}(a)(\bar{x}(a)+\epsilon \bar{c}(a)) d a$. Then $\hat{\lambda}(a)=k(a) \Lambda$. From the equations of (4.3), we obtain that

$$
\begin{aligned}
\bar{\ell}(a)= & \Lambda \int_{0}^{a} e^{-\varpi(a-\eta)-\sigma(a-\eta)-\int_{\eta}^{a} b(\xi) d \xi} k(\eta) s^{0}(\eta) d \eta, \\
\bar{x}(a)= & \sigma \int_{0}^{a} e^{-\int_{\eta}^{a}\left(\varpi+\gamma_{1}+b(\xi)\right) d \xi} \bar{l}(\eta) d \eta \\
\bar{c}(a)= & \bar{c}(0) e^{-\int_{0}^{a}\left(\varpi+b(\xi) \omega \nu+\mu_{1}(\xi)+b(\xi)+\gamma_{2}(\xi)\right) d \xi} \\
& +\int_{0}^{a} e^{-\int_{\eta}^{a}\left(\varpi+b(\xi) \omega \nu+\mu_{1}(\xi)+b(\xi)+\gamma_{2}(\xi)\right) d \xi} q(\eta) \gamma_{1} \bar{x}(\eta) d \eta .
\end{aligned}
$$

Since the perinatal infection is about $11 \%$ of carrier mothers (Edmunds, Medley, and Nokes [7]), which is much smaller than the proportion of all infected individuals, in the rest of the discussion we make the following assumption.

Assumption 4.1. Assume that $\nu=0$; i.e., we ignore the proportion of those perinatally infected from carrier mothers.

Define

$$
\Phi(a):=\int_{0}^{a} e^{-\varpi(a-\eta)-\sigma(a-\eta)-\int_{\eta}^{a} b(\xi) d \xi} k(\eta) s^{0}(\eta) d \eta .
$$

Substituting (4.4) into (4.5), we have

$$
\bar{x}(a)=\Lambda \sigma \int_{0}^{a} e^{-\int_{\eta}^{a}\left(\varpi+\gamma_{1}+b(\xi)\right) d \xi} \Phi(\eta) d \eta .
$$

Under Assumption 4.1, we substitute (4.7) into (4.6) and obtain that

$$
\bar{c}(a)=\Lambda \sigma \gamma_{1} \int_{0}^{a} \int_{0}^{\eta} e^{-\varpi(a-\zeta)-\gamma_{1}(\eta-\zeta)-\int_{\eta}^{a}\left(\mu_{1}(\xi)+\gamma_{2}(\xi)\right) d \xi-\int_{\zeta}^{a} b(\xi) d \xi} q(\eta) \Phi(\zeta) d \zeta d \eta .
$$

We further substitute (4.7) and (4.8) into the expression of $\lambda$ and have that

$$
\begin{aligned}
& \Lambda=\Lambda \sigma \int_{0}^{a_{+}} \tilde{\beta}(a) \int_{0}^{a} e^{-\int_{\eta}^{a}\left(\varpi+\gamma_{1}+b(\xi)\right) d \xi} \Phi(\eta) d \eta d a \\
& +\epsilon \Lambda \sigma \gamma_{1} \int_{0}^{a_{+}} \tilde{\beta}(a) \int_{0}^{a} \int_{0}^{\eta} e^{-\varpi(a-\zeta)-\gamma_{1}(\eta-\zeta)-\int_{\eta}^{a}\left(\mu_{1}(\xi)+\gamma_{2}(\xi)\right) d \xi-\int_{\zeta}^{a} b(\xi) d \xi} q(\eta) \Phi(\zeta) d \zeta d \eta d a,
\end{aligned}
$$

which yields the characteristic equation

$$
\begin{aligned}
1= & \sigma \int_{0}^{a_{+}} \tilde{\beta}(a) \int_{0}^{a}\left[e^{-\int_{\eta}^{a}\left(\varpi+\gamma_{1}+b(\xi)\right) d \xi} \Phi(\eta)\right. \\
& \left.+\epsilon \gamma_{1} \int_{0}^{\eta} e^{-\varpi(a-\zeta)-\gamma_{1}(\eta-\zeta)-\int_{\eta}^{a}\left(\mu_{1}(\xi)+\gamma_{2}(\xi)\right) d \xi-\int_{\zeta}^{a} b(\xi) d \xi} q(\eta) \Phi(\zeta) d \zeta\right] d \eta d a .
\end{aligned}
$$

Denote the right-hand side of (4.9) by $\tilde{F}(\varpi)$. We define the basic reproduction number (Diekmann, Heesterbeek, and Metz [6]) as $R_{0}=\tilde{F}(0)$, or explicitly as

$$
\begin{aligned}
R_{0}= & \sigma \int_{0}^{a_{+}} \tilde{\beta}(a) \int_{0}^{a}\left[e^{-\int_{\eta}^{a}\left(\gamma_{1}+b(\xi)\right) d \xi} \tilde{\Phi}(\eta)\right. \\
& \left.+\epsilon \gamma_{1} \int_{0}^{\eta} e^{-\gamma_{1}(\eta-\zeta)-\int_{\eta}^{a}\left(\mu_{1}(\xi)+\gamma_{2}(\xi)\right) d \xi-\int_{\zeta}^{a} b(\xi) d \xi} q(\eta) \tilde{\Phi}(\zeta) d \zeta\right] d \eta d a,
\end{aligned}
$$

Copyright $@$ by SIAM. Unauthorized reproduction of this article is prohibited. 
where

$$
\tilde{\Phi}(a)=\int_{0}^{a} e^{-\sigma(a-\eta)-\int_{\eta}^{a} b(\xi) d \xi} k(\eta) s^{0}(\eta) d \eta .
$$

We have the following result on the local stability of the disease-free steady state.

TheOREM 4.2. Under Assumption 4.1, the disease-free steady state $E^{0}$ is locally asymptotically stable if $R_{0}<1$ and unstable if $R_{0}>1$.

Proof. The function $\tilde{F}(\varpi)$ is a decreasing and continuous function of $\varpi$ which approaches $\infty$ when $\varpi \rightarrow-\infty$ and zero when $\varpi \rightarrow \infty$. Therefore, the characteristic equation (4.9) has a unique real solution $\varpi^{*}$. If $\tilde{F}(0)<1$, or, equivalently, $R_{0}<1$, then the real solution of $\tilde{F}(\varpi)=1$ satisfies $\varpi^{*}<0$ and the unique real eigenvalue is negative.

Furthermore, we consider other solutions of (4.9). Let $\varpi=\alpha+\gamma i$. Since $\operatorname{Re} e^{\varpi} \leq$ $e^{\operatorname{Re} \varpi}$, from (4.9) we know that $\operatorname{Re} \tilde{F}(\varpi)=1, \operatorname{Im} \tilde{F}(\varpi)=0$, and $1 \leq \tilde{F}(\operatorname{Re} \varpi)$. We immediately obtain that $\operatorname{Re} \varpi \leq \varpi^{*}$ since $\tilde{F}(\varpi)$ is a decreasing function of $\varpi$. That is, all complex solutions of (4.9) have real parts smaller than $\varpi^{*}$, and therefore are negative. Hence, the disease-free steady state is locally asymptotically stable if $R_{0}>1$. On the other hand, if $\tilde{F}(0)>1$, or, equivalently, $R_{0}>1$, the unique real solution of (4.9) is positive, and the disease-free steady state is unstable.

5. Disease-endemic steady states. In this section, we discuss the existence and stability of the disease-endemic steady states under Assumption 4.1. Though many people die from HBV-related diseases every year, the number of deaths directly related to HBV is small. For example, the reported mortality rate of HBV in China was $0.0464 \%$ in January 2009 (see [24]). Based on this observation, we make the following additional assumption.

Assumption 5.1. Assume that $\mu_{1}(a)=0$; i.e., we ignore deaths directly related to HBV.

We have the following result on the existence of the disease-endemic steady state.

THEOREM 5.2. If Assumptions 4.1 and 5.1 are satisfied and $R_{0}>1$, then there is a unique disease-endemic steady state $E^{*}=\left(s^{*}(a), l^{*}(a), x^{*}(a), c^{*}(a), v^{*}(a)\right)$ of system $(2.5)$.

Proof. Under Assumptions 4.1 and 5.1, the coordinates of $E^{*}$ are given by

$$
\begin{aligned}
s^{*}(a)= & \omega e^{-\int_{0}^{a}\left(b(\xi)+k(\xi) \Lambda^{*}+p(\xi)\right) d \xi} \\
& +\int_{0}^{a} e^{-\int_{\eta}^{a}\left(b(\xi)+k(\xi) \Lambda^{*}+p(\xi)\right) d \xi}\left[b(\eta) \omega+\psi v^{*}(\eta)\right] d \eta, \\
\ell^{*}(a)= & \int_{0}^{a} e^{-\sigma(a-\eta)-\int_{\eta}^{a} b(\xi) d \xi} k(\eta) \Lambda^{*} s^{*}(\eta) d \eta, \\
x^{*}(a)= & \sigma \int_{0}^{a} e^{-\gamma_{1}(a-\eta)-\int_{\eta}^{a} b(\xi) d \xi} \ell^{*}(\eta) d \eta, \\
c^{*}(a)= & \int_{0}^{a} e^{-\int_{\eta}^{a}\left(\gamma_{2}(\xi)+b(\xi)\right) d \xi} q(\eta) \gamma_{1} x^{*}(\eta) d \eta, \\
v^{*}(a)= & (1-\omega) e^{-\int_{0}^{a}(\psi+b(\xi)) d \xi} \\
& +\int_{0}^{a} e^{-\int_{\eta}^{a}(\psi+b(\xi)) d \xi}\left(b(\eta)(1-\omega)+p(\eta) s^{*}(\eta)\right) d \eta,
\end{aligned}
$$

where $\Lambda^{*}=\int_{0}^{a_{+}} \tilde{\beta}(a)\left(x^{*}(a)+\epsilon c^{*}(a)\right) d a$. 
Substituting (5.5) into (5.1), we obtain

$$
s^{*}(a)=G(a)+\int_{0}^{a} K\left(a, \varsigma, \Lambda^{*}\right) s^{*}(\varsigma) d \varsigma,
$$

where

$$
\begin{aligned}
G(a)= & \omega e^{-\int_{0}^{a}\left(b(\xi)+k(\xi) \Lambda^{*}+p(\xi)\right) d \xi}+\int_{0}^{a} e^{b(\xi)+k(\xi) \Lambda^{*}+p(\xi) d \xi}[b(\eta) \omega \\
& \left.+\psi(1-\omega)\left(e^{-\int_{0}^{\eta}(\psi+b(\xi)) d \xi}+\int_{0}^{\eta} e^{-\int_{\varsigma}^{\eta}(\psi+b(\xi)) d \xi} b(\varsigma) d \varsigma\right)\right] d \eta, \\
K\left(a, \varsigma, \Lambda^{*}\right)= & \psi p(\varsigma) \int_{\varsigma}^{a} e^{-\int_{\eta}^{a}\left(b(\xi)+k(\xi) \Lambda^{*}+p(\xi)\right) d \xi-\int_{\varsigma}^{\eta}(\psi+b(\xi)) d \xi} d \eta .
\end{aligned}
$$

Then for every $\Lambda^{*}$ there is a unique solution $s^{*}\left(a, \Lambda^{*}\right)$ which depends continuously on $\Lambda^{*}$.

Substituting (5.2) into (5.3), we obtain

$$
x^{*}(a)=\Lambda^{*} \sigma \int_{0}^{a} \int_{0}^{\eta} e^{-\gamma_{1}(a-\eta)-\sigma(\eta-\varsigma)-\int_{\varsigma}^{a} b(\xi) d \xi} k(\varsigma) s^{*}(\varsigma) d \varsigma d \eta,
$$

which together with (5.4) implies

$$
c^{*}(a)=\gamma_{1} \sigma \Lambda^{*} \int_{0}^{a} \int_{0}^{\eta} \int_{0}^{\varsigma} e^{-\int_{\eta}^{a} \gamma_{2}(\varsigma) d \varsigma-\gamma_{1}(\eta-\varsigma)-\int_{\rho}^{a} b(\xi) d \xi-\sigma(\varsigma-\rho)} q(\eta) k(\rho) s^{*}(\rho) d \rho d \varsigma d \eta .
$$

Substituting the expressions of $x^{*}(a)$ and $c^{*}(a)$ into the expression of $\Lambda^{*}$ and canceling $\Lambda^{*}$, we get

$$
\begin{aligned}
1= & \int_{0}^{a_{+}} \sigma \tilde{\beta}(a) \int_{0}^{a} \int_{0}^{\eta}\left\{e^{-\gamma_{1}(a-\eta)-\sigma(\eta-\varsigma)-\int_{\varsigma}^{a} b(\xi) d \xi} k(\varsigma) s^{*}(\varsigma)\right. \\
& \left.+\epsilon \gamma_{1} \int_{0}^{\varsigma} e^{-\int_{\eta}^{a} \gamma_{2}(\xi) d \xi-\gamma_{1}(\eta-\varsigma)-\sigma(\varsigma-\rho)-\int_{\rho}^{a} b(\xi) d \xi} q(\eta) k(\rho) s^{*}(\rho) d \rho\right\} d \varsigma d \eta d a .
\end{aligned}
$$

Denote the right-hand side of (5.7) by $H\left(\Lambda^{*}\right)$. Then $H\left(\Lambda^{*}\right)$ is a continuous decreasing function of $\Lambda^{*}$. Moreover, $s^{*}(a, 0)$ is $s^{0}$, which is the disease-free steady state. Hence, $H(0)=R_{0}$. That is, $R_{0}>1$ implies $H(0)>1$. On the other hand, for $\Lambda^{*}>0$,

$$
H\left(\Lambda^{*}\right)=\frac{1}{\Lambda^{*}} \int_{0}^{a_{+}} \tilde{\beta}(a)\left[x^{*}(a)+\epsilon c^{*}(a)\right] d a<\frac{1}{\Lambda^{*}} \int_{0}^{a_{+}} \tilde{\beta}(a) d a .
$$

The right-hand side of (5.8) approaches zero as $\Lambda^{*}$ approaches infinity. Therefore, $H\left(\Lambda^{*}\right)=1$ has a unique solution in $(0,+\infty)$. Furthermore, if $\Lambda^{*} \geq \int_{0}^{a_{+}} \tilde{\beta}(a) d a$, then $H\left(\Lambda^{*}\right)<1$. Hence, (5.7) has a unique solution in $\left(0, \int_{0}^{a_{+}} \tilde{\beta}(a) d a\right)$ and a diseaseendemic steady state exists.

To consider the linear stability of the disease-endemic steady state, let $\hat{s}(a, t)$, $\hat{\ell}(a, t), \hat{x}(a, t), \hat{c}(a, t)$, and $\hat{v}(a, t)$ be the perturbations of $s^{*}(a), \ell^{*}(a), x^{*}(a), c^{*}(a)$, and $v^{*}(a)$, respectively. Consider the exponential solutions of the system for the perturbations

$$
\begin{aligned}
& \hat{s}(a, t)=e^{\varrho t} \bar{s}(a), \quad \hat{\ell}(a, t)=e^{\varrho t} \bar{\ell}(a), \quad \hat{x}(a, t)=e^{\varrho t} \bar{x}(a), \\
& \hat{c}(a, t)=e^{\varrho t} \bar{c}(a), \quad \hat{v}(a, t)=e^{\varrho t} \bar{v}(a) .
\end{aligned}
$$

Copyright $\odot$ by SIAM. Unauthorized reproduction of this article is prohibited. 
We have

$$
\begin{aligned}
& \frac{d \bar{s}}{d a}=-\varrho \bar{s}(a)+\psi \bar{v}(a)-b(a) \bar{s}(a)-k(a) \bar{\Lambda} s^{*}(a)-k(a) \Lambda^{*} \bar{s}(a)-p(a) \bar{s}(a), \\
& \frac{d \bar{\ell}}{d a}=-\varrho \bar{\ell}(a)+k(a) \bar{\Lambda} s^{*}(a)+k(a) \Lambda^{*} \bar{s}(a)-(\sigma+b(a)) \bar{\ell}(a), \\
& \frac{d \bar{x}}{d a}=-\varrho \bar{x}(a)+\sigma \bar{\ell}(a)-\left(\gamma_{1}+b(a)\right) \bar{x}(a), \\
& \frac{d \bar{c}}{d a}=-\varrho \bar{c}(a)+q(a) \gamma_{1} \bar{x}(a)-\left(b(a)+\gamma_{2}(a)\right) \bar{c}(a), \\
& \frac{d \bar{v}}{d a}=-\varrho \bar{v}(a)+p(a) \bar{s}(a)-(\psi+b(a)) \bar{v}(a),
\end{aligned}
$$

with the initial conditions

$$
\bar{s}(0)=\bar{\ell}(0)=\bar{x}(0)=\bar{c}(0)=\bar{v}(0)=0,
$$

where

$$
\bar{\Lambda}=\int_{0}^{a_{+}} \tilde{\beta}(a)(\bar{x}(a)+\epsilon \bar{c}(a)) d a .
$$

Assume that $\bar{\Lambda} \neq 0$. Let $s=\bar{s} / \bar{\Lambda}, \ell=\bar{\ell} / \bar{\Lambda}, x=\bar{x} / \bar{\Lambda}, c=\bar{c} / \bar{\Lambda}$, and $v=\bar{v} / \bar{\Lambda}$. Then system (5.9) becomes

$$
\begin{aligned}
& \frac{d s}{d a}=-\varrho s(a)+\psi v(a)-b(a) s(a)-k(a) s^{*}(a)-k(a) \Lambda^{*} s(a)-p(a) s(a), \\
& \frac{d \ell}{d a}=-\varrho \ell(a)+k(a) s^{*}(a)+k(a) \Lambda^{*} s(a)-(\sigma+b(a)) \ell(a), \\
& \frac{d x}{d a}=-\varrho x(a)+\sigma \ell(a)-\left(\gamma_{1}+b(a)\right) x(a), \\
& \frac{d c}{d a}=-\varrho c(a)+q(a) \gamma_{1} x(a)-\left(b(a)+\gamma_{2}(a)\right) c(a), \\
& \frac{d v}{d a}=-\varrho v(a)+p(a) s(a)-(\psi+b(a)) v(a),
\end{aligned}
$$

with the initial conditions

$$
s(0)=\ell(0)=x(0)=c(0)=v(0)=0 .
$$

Then the solution of (5.10) satisfies the equation

$$
1=\int_{0}^{a_{+}} \tilde{\beta}(a)(x(a)+\epsilon c(a)) d a .
$$

Denote the right-hand side of $(5.11)$ by $\bar{H}(\varrho)$. Then it satisfies the following proposition.

Proposition 5.3. (1) $\bar{H}(\varrho)$ is a decreasing function of $\varrho$, which tends to zero as $\varrho \rightarrow \infty ;(2) \bar{H}(0)<1$.

Copyright (c) by SIAM. Unauthorized reproduction of this article is prohibited. 
Proof. From (5.10), we have

$$
\begin{aligned}
& s(a)=\int_{0}^{a} e^{-\int_{\eta}^{a}\left(\varrho+b(\xi)+k(\xi) \Lambda^{*}+p(\xi)\right) d \xi}\left(\psi v(\eta)-k(\eta) s^{*}(\eta)\right) d \eta, \\
& \ell(a)=\int_{0}^{a} e^{-\int_{\eta}^{a}(\varrho+b(\xi)+\sigma) d \xi}\left(k(\eta) s^{*}(\eta)+k(\eta) \Lambda^{*} s(\eta)\right) d \eta, \\
& x(a)=\int_{0}^{a} e^{-\int_{\eta}^{a}\left(\varrho+b(\xi)+\gamma_{1}\right) d \xi} \sigma \ell(\eta) d \eta, \\
& c(a)=\int_{0}^{a} e^{-\int_{\eta}^{a}\left(\varrho+b(\xi)+\gamma_{2}(\xi)\right) d \xi} q(\eta) \gamma_{1} x(\eta) d \eta, \\
& v(a)=\int_{0}^{a} e^{-\int_{\eta}^{a}(\varrho+\psi+b(\xi)) d \xi} p(\eta) s(\eta) d \eta .
\end{aligned}
$$

Substituting (5.14) into (5.15) and using the expression of $\bar{H}(\varrho)$, we get

$$
\begin{aligned}
\bar{H}(\varrho)= & \sigma \int_{0}^{a_{+}} \tilde{\beta}(a) \int_{0}^{a} e^{-\varrho(a-\eta)-\int_{\eta}^{a} b(\xi) d \xi}\left[e^{-\gamma_{1}(a-\eta)} \ell(\eta)\right. \\
& \left.+\epsilon \gamma_{1} q(\eta) e^{-\int_{\eta}^{a} \gamma_{2}(\xi) d \xi} \int_{0}^{\eta} e^{-\varrho(\eta-\varsigma)-\gamma_{1}(\eta-\varsigma)-\int_{\varsigma}^{\eta} b(\xi) d \xi} \ell(\varsigma) d \varsigma\right] d \eta .
\end{aligned}
$$

From (5.11) we have $x(a)+\epsilon c(a)>0$, which implies that $\ell(a)>0$. Therefore, from (5.17) we know that $\bar{H}(\varrho)$ decreases exponentially in $\varrho$ and $\bar{H}(\varrho)$ approaches 0 as $\varrho$ tends to infinity.

Substituting (5.13) into (5.17) and taking $\varrho=0$, we have

$$
\begin{aligned}
\bar{H}(0)= & \sigma \int_{0}^{a_{+}} \tilde{\beta}(a) \int_{0}^{a} e^{-\int_{\eta}^{a} b(\xi) d \xi}\left[e^{-\gamma_{1}(a-\eta)-\sigma(\eta-\varsigma)} k(\varsigma) s^{*}(\varsigma)\right. \\
& \left.+\epsilon \gamma_{1} e^{-\gamma_{1}(\eta-\varsigma)-\int_{\eta}^{a} \gamma_{2}(\xi) d \xi} q(\eta) \int_{0}^{\varsigma} e^{-\sigma(\varsigma-\tau)-\int_{\tau}^{\varsigma} b(\xi) d \xi} k(\tau) s^{*}(\tau) d \tau\right] d \varsigma d \eta d a \\
& +\Lambda^{*} \sigma \int_{0}^{a_{+}} \tilde{\beta}(a) \int_{0}^{a} \int_{0}^{\eta} e^{-\int_{\varsigma}^{a} b(\xi) d \xi}\left[e^{-\gamma_{1}(a-\eta)-\sigma(\eta-\varsigma)} k(\varsigma) s(\varsigma)\right. \\
& \left.+\epsilon \gamma_{1} e^{-\gamma_{1}(\eta-\varsigma)-\int_{\eta}^{a} \gamma_{2}(\xi) d \xi} q(\eta) \int_{0}^{\varsigma} e^{-\sigma(\varsigma-\tau)-\int_{\tau}^{\varsigma} b(\xi) d \xi} k(\tau) s(\tau) d \tau\right] d \varsigma d \eta d a .
\end{aligned}
$$

Equation (5.7) implies that the first integral of (5.18) is equal to one. Furthermore, let $y(a)=s(a)+\ell(a)+x(a)+c(a)+v(a)$. From (5.10), we have

$$
\frac{d y}{d a}=-\varrho y(a)-b(a) y(a)-(1-q(a)) \gamma_{1} x(a)-\gamma_{2}(a) c(a),
$$

which has a solution

$$
y(a)=-\int_{0}^{a} e^{-\int_{\eta}^{a}(\omega+b(\xi)) d \xi}\left((1-q(\eta)) \gamma_{1} x(\eta)+\gamma_{2}(\eta) c(\eta)\right) d \eta .
$$

By $x(a)+\epsilon c(a)>0$ and (5.15), we know that $x(a)>0$ and $c(a)>0$. Then (5.20) implies that $y(a)<0$. Moreover, we have $\ell(a)>0$ from (5.14). Thus, $s(a)+v(a)<0$. On the other hand, (5.16) implies that $v(a)$ has the same sign as $s(a)$. Therefore, $s(a)<0$. Finally, from $(5.18)$ we obtain that $\bar{H}(0)<1$.

Copyright (c) by SIAM. Unauthorized reproduction of this article is prohibited. 
Consequently, $\bar{H}(\varrho)=1$ has a unique real solution which is negative, and all complex solutions have real parts smaller than the unique real solution. Therefore, we obtain the following result on the stability of the disease-endemic steady state.

TheOREM 5.4. If Assumptions 4.1 and 5.1 are satisfied and $R_{0}>1$, then the disease-endemic steady state $E^{*}$ of system (3.1) is stable.

6. Numerical simulations. Since hepatitis B infection is most endemic in China and the model is based on our previous study on HBV transmission dynamics in China, our numerical simulations are based on some main parameters used or derived in Zhao, Xu, and $\mathrm{Lu}$ [31] and Zou, Zhang, and Ruan [32] for HBV infection in China.

We first have the parameter $\lambda(a, 0)$ given by

$\lambda(a, 0)=\left\{\begin{array}{l}0.13074116-0.01362531 a+0.00046463 a^{2}-0.00000489 a^{3}, 0 \leq a \leq 47.5, \\ \lambda(47.5,0), a>47.5 .\end{array}\right.$

The probability $q(a)$ that an individual fails to clear an acute infection and develops to carrier state is

$$
q(a)=0.176501 \exp (-0.787711 a)+0.02116
$$

The remaining parameters are given in Table 1.

TABLE 1

Parameter values used in numerical simulations.

\begin{tabular}{llll}
\hline Parameter & Interpretation & Value & Reference \\
\hline$b$ & Birth rate & 0.0121 & {$[24]$} \\
$\epsilon$ & Reduced transmission rate & 0.16 & {$[7]$} \\
$\sigma$ & Rate moving from latent to acute & 6 per yr & {$[7]$} \\
$\gamma_{1}$ & Rate moving from acute to carrier & 4 per yr & {$[7]$} \\
$\gamma_{2}$ & Rate moving from carrier to immune & 0.025 per yr & {$[7]$} \\
$\psi$ & Rate of waning vaccine-induced immunity & 0.1 & {$[8]$} \\
\hline
\end{tabular}

Using a constant $p$ for $p(a)$, we simulate the properties of disease-endemic steady states. In Figure 2, the solid curves represent the states with $p=0.4$, while the dash curves represent the states with $p=0.3$. It is obvious from Figure 2 that the maximum values of acute infections and carriers with $p=0.3$ are larger than those with $p=0.4$.

To control HBV infection, we would like to see what parameters can reduce the basic reproduction number $R_{0}$ defined by (4.10). From Figure 3(a) we can see that for fixed $p, R_{0}$ decreases when $1-\omega$ increases. Similarly, in Figure 3(b) when $\omega$ is fixed, $R_{0}$ decreases if $p$ increases. Consequently, $R_{0}$ decreases as both $1-\omega$ and $p$ increase (Figure 3(c)). Thus, the optimal control strategy is a combination of immunization of newborns and retroactive immunization of susceptible adults.

Using equivalent time steps in the age and time directions, we choose 50 steps per year and solve the system numerically. Figure 4 presents the age distribution of the proportion of carriers $c(a, t)$, where the initial value $c(a, 0)$ is constructed based on the data in Table 3 of $\mathrm{Zhao}, \mathrm{Xu}$, and $\mathrm{Lu}$ [31].

7. Discussion. The transmission of HBV is characterized by two age-dependent processes: the per-capita rate of infection and the risk of becoming a carrier (Edmunds, Medley, and Nokes [7]). Taking age-dependent heterogeneity into consideration, several groups of researchers have proposed age-structured models to study the 
(a)

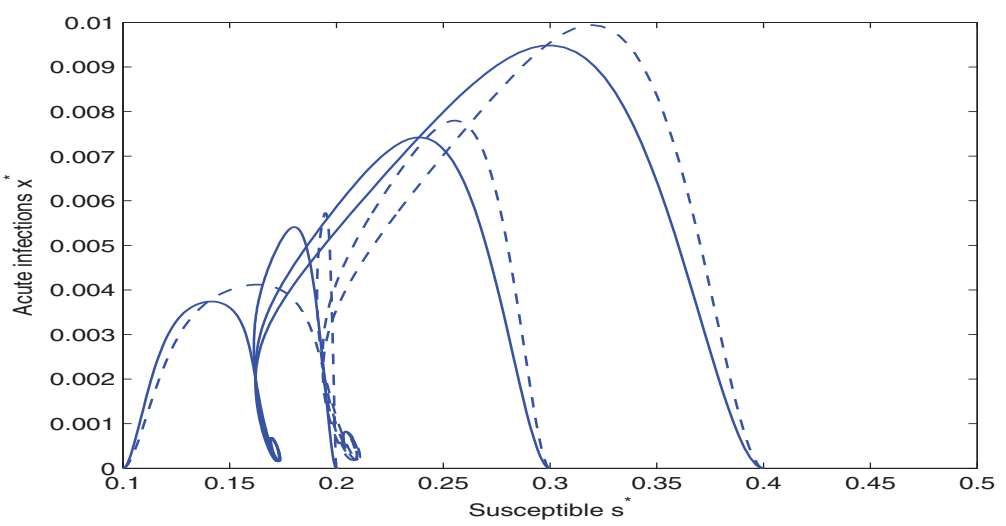

(b)

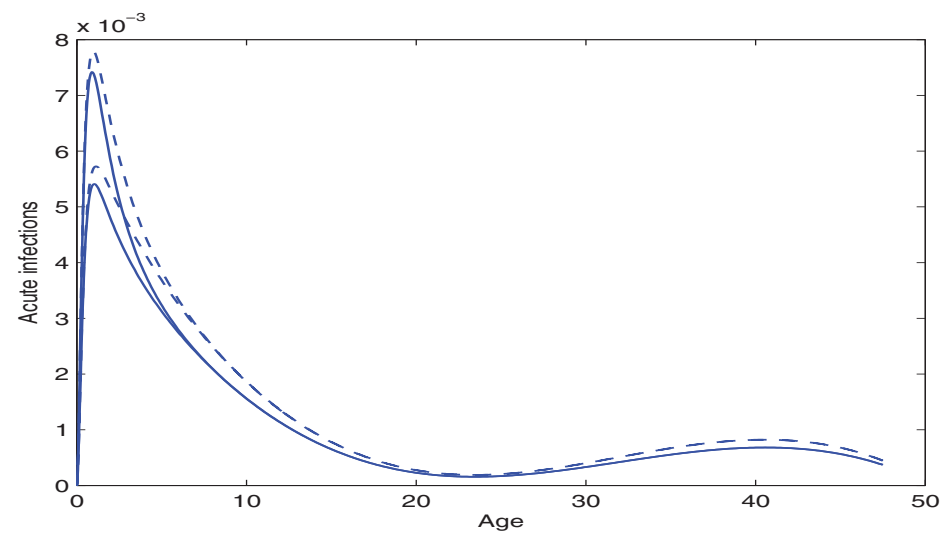

(c)

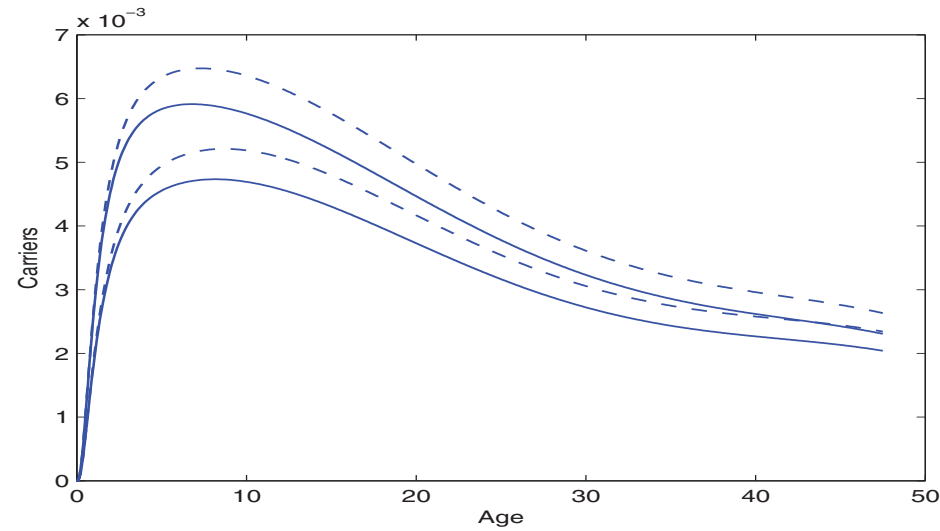

FIG. 2. (a) The disease-endemic steady states of susceptible and acute infections. (b) The predicted proportion of acute infections by age. (c) The predicted proportion of carriers by age.

Copyright (C) by SIAM. Unauthorized reproduction of this article is prohibited. 
(a)

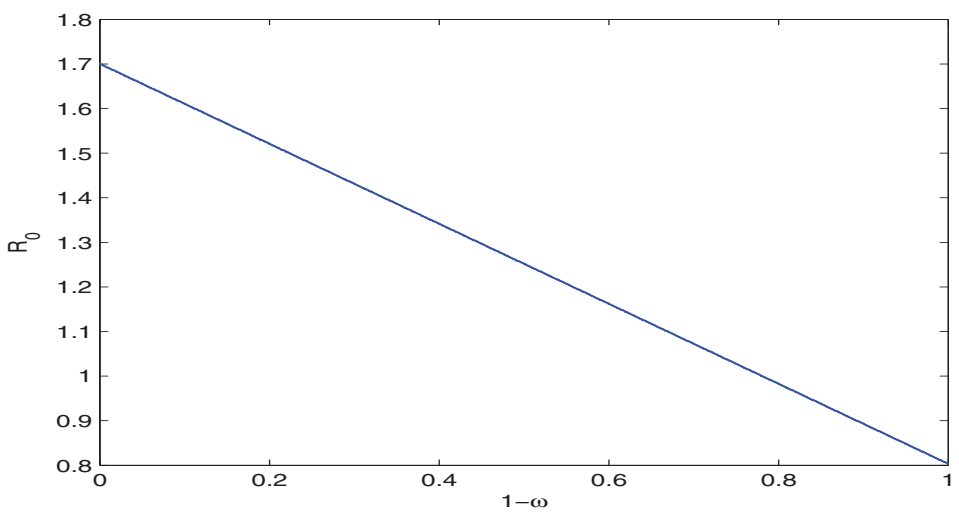

(b)

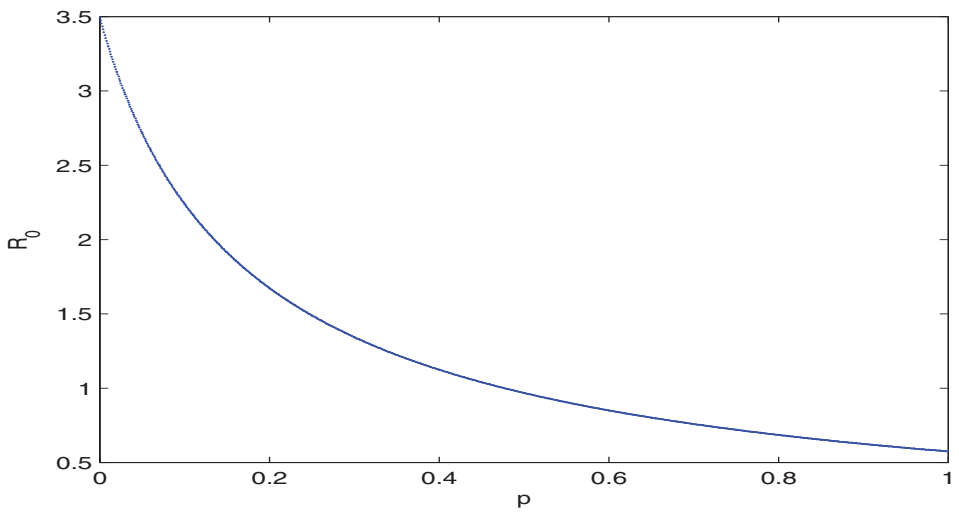

(c)

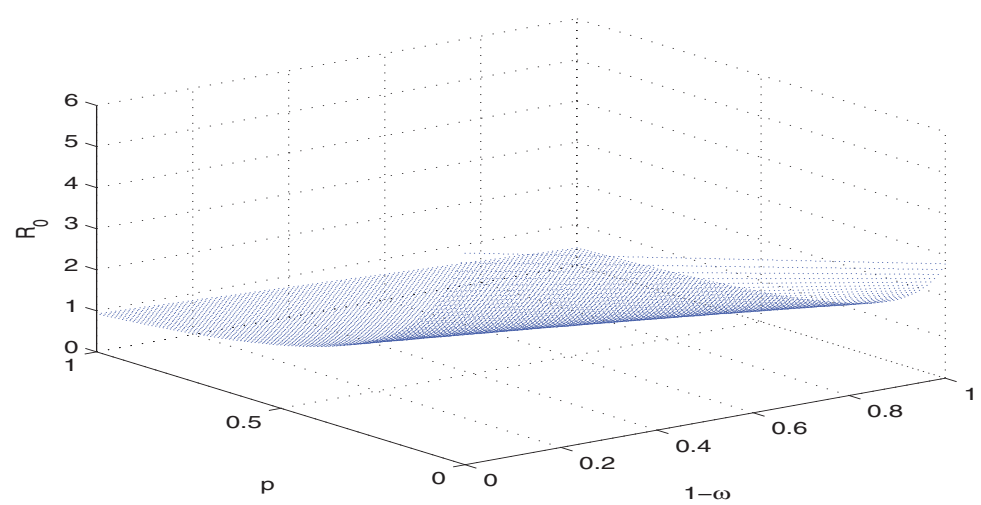

FIG. 3. The plots of the basic reproduction number $R_{0}$ in terms of some parameters. (a) $R_{0}$ in terms of $1-\omega$. (b) $R_{0}$ as a function of $p$. (c) $R_{0}$ in terms of $1-\omega$ and $p$.

Copyright (c) by SIAM. Unauthorized reproduction of this article is prohibited. 


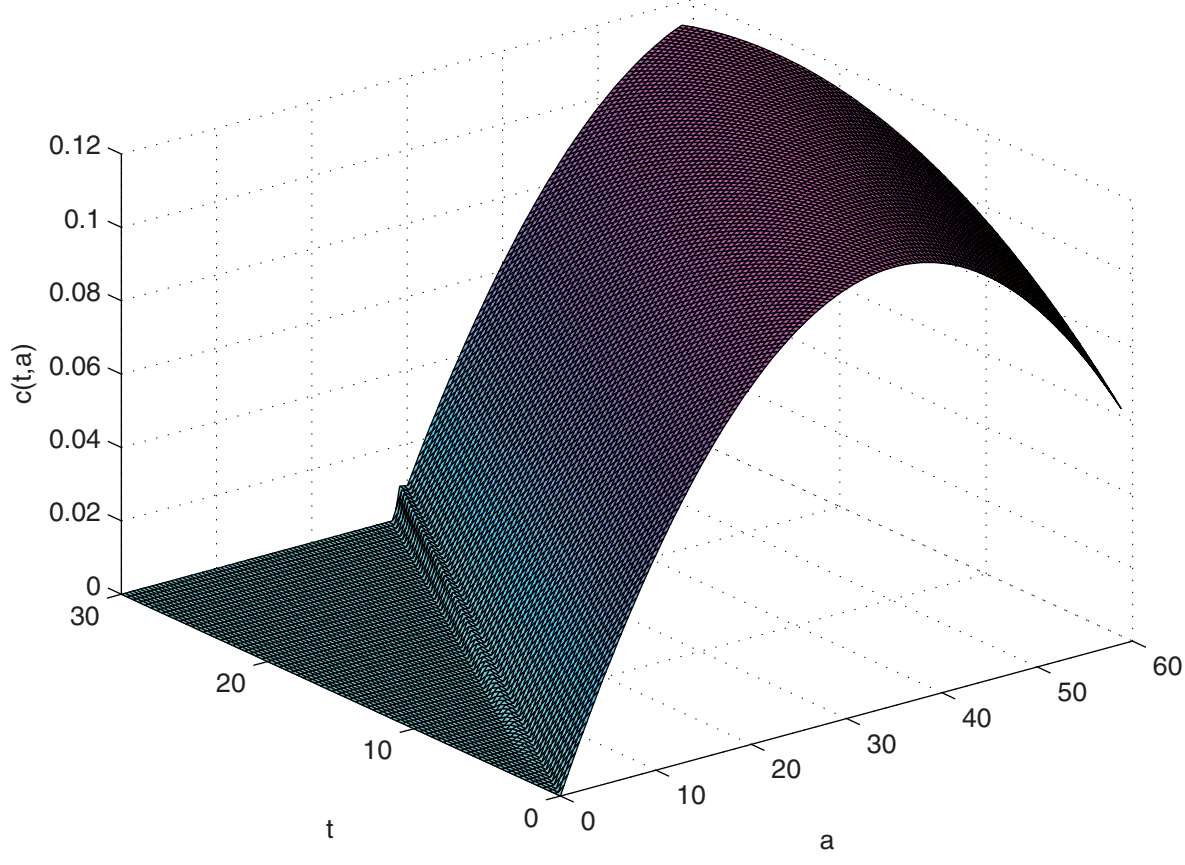

FIG. 4. The age distribution of the carriers $c(a, t)$.

transmission dynamics of HBV; see Edmunds et al. [9], McLean and Blumberg [21], Zhao, $\mathrm{Xu}$, and $\mathrm{Lu}$ [31], etc. Some of these models have also been used to evaluate the effectiveness of the vaccination program in newborns in some countries. For example, Zhao, $\mathrm{Xu}$, and $\mathrm{Lu}$ [31] used an age-structured model to predict the transmission dynamics of $\mathrm{HBV}$ and to evaluate the long-term effectiveness of the vaccination program in China. Their results suggest that HBV infection in China can be controlled in just one generation and eventually eliminated if all infants are immunized throughout the country, especially in poor rural areas. However, achieving such a high vaccination rate for infants in countries such as China is almost impossible. In fact, despite an effective national vaccination program for newborn babies since the 1990s, which has reduced chronic $\mathrm{HBV}$ infection in children, the incidence of hepatitis B in China is still increasing (Zou, Zhang, and Ruan [32]).

Mass vaccination in infants increases the average age of infection in unimmunized individuals and shifts the average age at infection to older age groups (Edmunds, Medley, and Nokes [7]). This indicates that mass vaccination in infants might be not enough to control the infection and eradicate the virus. Different immunization programs can be evaluated by considering the prevalence of carriers after the implementation of immunization. The specific character of HBV infection in China is that there is a large number of HBV carriers. In a previous study (Zou, Zhang, and Ruan [32]), we developed an ordinary differential equations model to study the transmission dynamics and control of HBV in mainland China, taking into account the character of the virus infection in the country. Based on our previous work [32], in this article we proposed an age-structured model for the transmission of HBV. We studied the existence and stability of the disease-free and disease-endemic steady states in terms of the basic reproduction number. The analytical results and numeri- 
cal simulations of the model suggest that the optimal control strategy is a combination of immunization of newborns and retroactive immunization of susceptible adults.

Acknowledgment. We would like to thank the referees for their helpful comments and valuable suggestions.

\section{REFERENCES}

[1] R. M. Anderson And R. M. May, Infectious Diseases of Humans: Dynamics and Control, Oxford University Press, Oxford, 1991.

[2] R. M. Anderson, R. M. May, And D. J. Nokes, Preliminary analyses of the predicted impacts of various vaccination strategies on the transmission of hepatitis B virus, in The Control of Hepatitis B: The Role of Prevention in Adolescence, D. L. Bennet, ed., Gower Medical Publishing, London, 1992, pp. 95-130.

[3] S. Busenberg, K. Cooke, And M. IAnnelli, Endemic thresholds and stability in a class of age-structured epidemics, SIAM J. Appl. Math., 48 (1988), pp. 1379-1395.

[4] S. N. Busenberg, M. Iannelli, and H. R. Thieme, Global behavior of an age-structured epidemic model, SIAM J. Math. Anal., 22 (1991), pp. 1065-1080.

[5] Centers for Disease Control and Prevention (CDC), The Pre-travel Consultation TravelRelated Vaccine-Preventable Diseases: Hepatitis B, in Travelers' Health-Yellow Book, Chapter 2, http://wwwn.cdc.gov/travel/yellowbook/2010/chapter-2/hepatitis-b.aspx.

[6] O. Diekmann, J. A. P. Heesterbeek, and J. A. J. Metz, On the definition and the computation of the basic reproduction ratio $R_{0}$ in models for infectious diseases in heterogeneous populations, J. Math. Biol., 28 (1990), pp. 365-382.

[7] W. J. Edmunds, G. F. Medley, And D. J. Nokes, The transmission dynamics and control of hepatitis B virus in the Gambia, Stat. Med., 15 (1996), pp. 2215-2233.

[8] W. J. Edmunds, G. F. Medley, And D. J. Nokes, Vaccination against hepatitis B virus in highly endemic area: Waning vaccine-induced immunity and the need for booster doses, Trans. R. Soc. Trop. Med. Hyg., 90 (1996), pp. 436-440.

[9] W. J. Edmunds, G. F. Medley, D. J. Nokes, A. J. Hall, and H. C. Whittle, The influence of age on the development of the hepatitis B carrier state, Proc. R. Soc. Lond. B, 253 (1993), pp. 197-201.

[10] Z. Feng, W. Huang, and C. Castillo-Chavez, Global behavior of a multi-group SIS epidemic model with age structure, J. Differential Equations, 218 (2005), pp. 292-324.

[11] S. T. Goldstein, F. J. Zhou, S. C. Hadler, B. P. Bell, E. E. Mast, and H. S. Margolis, A mathematical model to estimate global hepatitis $B$ disease burden and vaccination impact, Int. J. Epidemiol., 34 (2005), pp. 1329-1339.

[12] D. Greenhalgh, Analytical results on the stability of age-structured recurrent epidemic models, IMA J. Math. Appl. Med. Biol., 4 (1987), pp. 109-144.

[13] D. Greenhalgh, Threshold and stability results for an epidemic model with age-structured meeting rate, IMA J. Math. Appl. Med. Biol., 5 (1988), pp. 81-100.

[14] M. IAnnelli, Mathematical Theory of Age-Structured Population Dynamics, Giadini Editori e Stampatori, Pisa, 1994.

[15] H. INABA, Threshold and stability results for an age-structured epidemic model, J. Math. Biol., 28 (1990), pp. 411-434.

[16] H. INABA, Mathematical analysis of an age-structured SIR epidemic model with vertical transmission, Discrete Contin. Dyn. Syst. Ser. B, 6 (2006), pp. 69-96.

[17] J. D. JiA AND H. ZhuAng, The overview of the seminar on chronic hepatitis B, Chin. J. Hepatol., 12 (2004), pp. 698-699.

[18] X.-Z. Li, G. Gupur, And G.-T. Zhu, Threshold and stability results for an age-structured SEIR epidemic model, Comput. Math. Appl., 42 (2001), pp. 883-907.

[19] G.-T. Liu, C.-W. Si, Q.-H. Wang, L.-H. Chen, H.-S. Chen, D.-Z. Xu, L.-X. Zhang, B.-E. WANG, L.-T. WANG, AND Y. LI, Comments on the prevention and research of chronic hepatitis in China, Natl. Med. J. China, 82 (2002), pp. 74-76.

[20] M. Martcheva and G. CRispino-O'Connell, The transmission of meningococcal infection: A mathematical study, J. Math. Anal. Appl., 283 (2003), pp. 251-275.

[21] A. R. McLean and B. S. Blumberg, Modelling the impact of mass vaccination against hepatitis B. I. Model formulation and parameter estimation, Proc. R. Soc. Lond. B, 256 (1994), pp. $7-15$.

[22] A. McKendrick, Applications of mathematics to medical problems, Proc. Edinb. Math. Soc., 44 (1926), pp. 98-130. 
[23] G. F. Medley, N. A. Lindop, W. J. Edmunds, And D. J. NoKes, Hepatitis-B virus endemicity: Heterogeneity, catastrophic dynamics and control, Nat. Med., 7 (2001), pp. 619-624.

[24] Ministry of Health of the People's Republic of China (MOHC), 2009, http://www.moh. gov.cn/publicfiles//business/htmlfiles/zwgkzt/pyq/list.htm.

[25] F. Sharpe And A. LotKa, A problem in age-distribution, Philos. Mag., 6 (1911), pp. 435-438.

[26] C. W. Shepard, E. P. Simard, L. Finelli, A. E. Fiore, and B. P. Bell, Hepatitis B virus infection: Epidemiology and vaccination, Epidemiol. Rev., 28 (2006), pp. 112-125.

[27] G. F. WebB, Population models structured by age, size, and spatial position, in Structured Population Models in Biology and Epidemiology, P. Magal and S. Ruan, eds., Lecture Notes in Math. 1936, Springer-Verlag, Berlin, New York, 2008, pp. 1-49.

[28] J. R. Williams, D. J. Nokes, G. F. Medley, and R. M. Anderson, The transmission dynamics of hepatitis $B$ in the UK: A mathematical model for evaluating costs and effectiveness of immunization programmes, Epidemiol. Infect., 116 (1996), pp. 71-89.

[29] World Health Organization (WHO), Expanded programme on immunization: Global Advisory Group, Part I, Weekly Epidemiological Record, 67 (1992), pp. 11-19.

[30] World Health Organization (WHO), Hepatitis B, Revised August 2008, http://www.who. int/mediacentre/factsheets/fs204/en/index.html.

[31] S.-J. ZhaO, Z.-Y. XU, AND Y. LU, A mathematical model of hepatitis $B$ virus transmission and its application for vaccination strategy in China, Int. J. Epidemiol., 29 (2000), pp. $744-752$.

[32] L. Zou, W. Zhang, ANd S. RUAN, Modelling the transmission dynamics and control of Hepatitis $B$ virus in China, J. Theoret. Biol., 262 (2010), pp. 330-338.

Copyright (c) by SIAM. Unauthorized reproduction of this article is prohibited. 